\title{
昜Digital Publisher
}

ISSN 2588-0705

La contabilidad de costos como alternativa para la toma de decisiones en Instituciones de servicios de salud pública.

Cost accounting as an alternative for decision making in public health service institutions.

Karla Virginia Navarrete-Cedeño Pontificia Universidad Católica del Ecuador Sede Manabí

knavarrete2696@pucesm.edu.ec

Dayana Valdés-Pérez

Pontificia Universidad Católica del Ecuador Sede Manabí dvaldes@pucesm.edu.ec

doi.org/10.33386/593dp.2020.5-1.348

V5-N5-1 (sep) 2020, pp. 244-251- | Recibido: 01 de julio de 2020 - Aceptado: 24 de agosto de 2020 (1 ronda rev.) Edición especial 


\section{RESUMEN}

El costo es un indicador importante a considerar para alcanzar mejoras en el proceso de toma de decisiones administrativas, el artículo tiene como objetivo analizar el uso de las herramientas de contabilidad de costos en las decisiones operativas de los servicios integrales de unidades de salud pública, la investigación es de carácter cualitativo, se realizó un estudio analítico de los resultados obtenidos a través de una encuesta que se aplicó a los doce responsables de las distintas áreas administrativas de una institución de salud pública de segundo nivel de atención de la zona 4 Manabí-Santo Domingo, en el Ecuador, donde se pudo constatar el limitado conocimiento y aplicación del costo en la toma de decisiones, por tal razón se propone la adaptación de un procedimiento enfocado en este fin.

Palabras clave: contabilidad; costos; gestión; salud; toma de decisiones.

Cómo citar este artículo:

APA:

Navarrete, K., \& Valdés, D. (2020). La contabilidad de costos como alternativa para la toma de decisiones en Instituciones de servicios de salud pública. 593 Digital Publisher CEIT, 5(5-1), 244-251. https://doi. org/10.33386/593dp.2020.5-1.348

Descargar para Mendeley y Zotero

\section{ABSTRACT}

Cost is an important indicator to be considered in order to achieve improvements in the administrative decision making process. The objective of this article is to analyze the use of cost accounting tools in operational decisions of comprehensive services in public health units; the research is of a qualitative nature, and an analytical study was conducted on the results obtained through a survey applied to the twelve heads of the different administrative areas of a public health institution of second level of care in zone 4 Manabí-Santo Domingo, in Ecuador, where it was possible to confirm the limited knowledge and application of cost in decision-making.

Key words: accounting; costs; management; health; decisión making 


\section{Introducción}

Uno de los aspectos claves para alcanzar la excelencia en las empresas, es la elevación de la eficiencia con el fin de mejorar la gestión y perfeccionar su entorno financiero.

Desde que la contabilidad se comenzó a entender como una herramienta de planeación y control, se han creado nuevas metodologías que cubran la creciente demanda de información para así anticiparse a los simples hechos económicos.

El entorno empresarial actual impone la necesidad de que las unidades organizativas modifiquen la gestión de sus procesos y actividades, en este aspecto la contabilidad de gestión proporciona información relevante, adecuada y oportuna para la dirección, la misma que aporta en la toma de decisiones internas y en especial de control de costos.

El costo utilizado como instrumento de dirección debe facilitar la valoración de posibles decisiones a tomar, permitiendo la selección de aquella que brinde el mayor beneficio productivo con el mínimo de gastos, así como, reducir el riesgo de fracasos en situaciones coyunturales de mercado o acciones impostergables de carácter nacional.

A partir del año 2008 Ecuador reconoce en su Constitución el derecho a la salud con una visión sistemática, vinculándolo con el ejercicio de otros derechos. La Carta Magna del 2008 establece al Estado como garante del derecho a la salud a través de la formulación de políticas, planes y programas orientados a brindar acceso a servicios de promoción y atención integral bajo los principios de equidad, universalidad, solidaridad, interculturalidad, calidad, eficiencia, eficacia, precaución y bioética, con enfoque de género y generacional. (Constitución de la República del Ecuador, 2008). Para que el Estado cumpla con esta obligación que señala la Constitución, se ve en la necesidad de tener sostenibilidad financiera del sistema de salud, en los últimos años la exigencia de los usuarios en prestaciones ha desencadenado una creciente demanda de servicios de salud. Cabe observar que, en el año 2007, el gasto en salud llegó a 2,8 mil millones de dólares, mientras en el 2013 ese monto alcanzó los 5,2 mil millones de dólares (INEC, 2016).

Esta situación demanda la búsqueda de alternativas de administración eficiente de los recursos, el uso de estos de forma eficiente y eficaz debe ser la finalidad principal del sistema de gestión de cualquier entidad pública, desde el punto de vista financiero, es necesario la correcta utilización del presupuesto disponible, mediante métodos de costos que permitan determinar el valor final del servicio de salud que recibe la población.

Con la creación de la Red Integral de Salud y sus mecanismos de relacionamiento, determinados en el convenio marco entre instituciones prestadoras del subsistema público (Ministerio de Salud Pública, 2015), juntamente con el Tarifario Nacional de Prestaciones y la Norma vigente de reconocimiento económico, rige la compra de servicios entre los prestadores y el sistema público y privado, lo que ha permitido disminuir las barreras de accesos a la salud entre subsistemas.

La complejidad de la gestión hospitalaria se refleja entre otros aspectos, en la diversidad de recursos utilizados, de procesos, y la complejidad técnica para la producción de los servicios de salud; por ejemplo enfermos con un mismo problema de salud, por sus características individuales, pueden ser atendidos con diferentes estudios diagnósticos y tratamientos, lo cual implica, desde el punto de vista de los costos, distinto consumo de recursos: tecnológicos, humanos y materiales (Vargas \& Hernández, 2009).

Al activar la compra de servicio a prestadores externos públicos o privados, salvo escasas excepciones cuando es por motivo de falta de espacio físico justificado por la demanda en servicios de salud, no se valora el costo de oportunidad al elegir compra o hacer.

Desde el punto de vista administrativo la toma de decisiones constituye un proceso esencial dentro de cualquier organización, esto se refiere solo a una parte dentro del proceso, es necesario 
que existan varios cursos de acción alternativos porque de lo contrario la decisión se reduciría a desarrollar o no determinada acción. El proceso de toma de decisiones se refiere a todas las actividades necesarias desde identificar un problema hasta finalmente resolverlo poniendo en práctica la alternativa seleccionada; por lo tanto, está enmarcado en la solución de problemas donde se debe encontrar alternativas de solución.

\section{Método}

La presente investigación es de carácter cualitativo, se realizó un estudio analítico de los resultados obtenidos a través de una encuesta que se aplicó a los doce responsables de las distintas áreas administrativas que pueden hacer uso de estas herramientas para la toma decisiones, ellas son:

Gerencia hospitalaria, Dirección asistencial, Departamento financiero y de planificación, Seguimiento y evaluación de la gestión de una institución de salud pública de segundo nivel de atención de la zona 4 Manabí-Santo Domingo, correspondiendo la población con la muestra encuestada, con la finalidad de determinar el nivel de aplicación de herramientas y técnicas de contabilidad de costo y gestión para la toma de decisiones.

Para tabular las encuestas se utilizó la página online encuesta.

La metodología utilizada incluye los siguientes puntos:

1. Revisión del marco normativo de las Instituciones de Salud Pública del Ecuador.

2. Revisión de literatura sobre técnicas de contabilidad gerencial y de costos.

3. Revisión de información cualitativa y cuantitativa de las instituciones de salud pública de segundo nivel de atención en Portoviejo.

4. Procedimiento para la toma de decisiones adaptado del modelo propuesto por Garza R. (2007).

\section{Resultados}

Se presentan los resultados de la encuesta realizada a 12 funcionarios de una institución de salud pública, en la cual se evidencia un alto porcentaje de desconocimiento de las herramientas de contabilidad de costo o gestión, como se muestra en la figura 1.

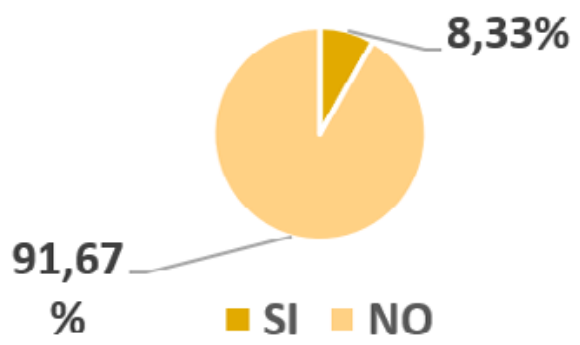

Figura 1. Conocimiento de las herramientas de contabilidad de costo

En la figura 2 se evidencia que el 66,67\% de los directivos desconocen sobre la aplicación de las herramientas de contabilidad de costo dentro de la institución de salud pública y por ende no las aplican para la toma de decisiones.

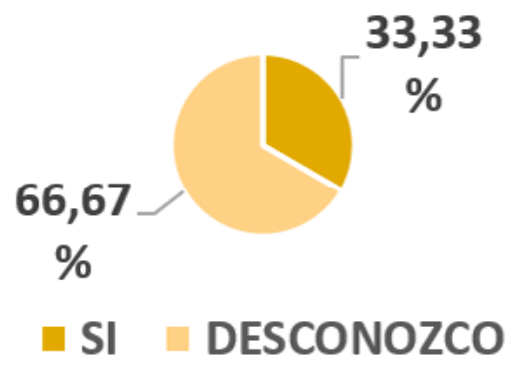

Figura 2. Conocimiento del uso de herramientas de contabilidad de costo por parte de los directivos

En la figura 3 se muestra que la mayoría de encuestados reconocen la importancia de la aplicación de herramientas de contabilidad de costos en el sector de la salud pública. 


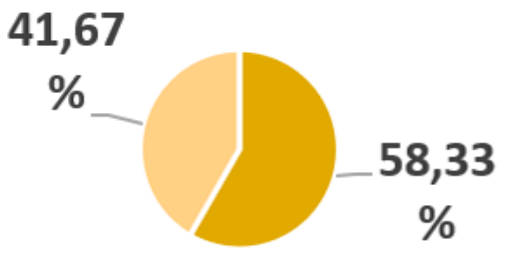

SI DESCONOZCO

Figura 3. Importancia de la aplicación de herramientas de contabilidad de costo

Los directivos de la Institución de salud pública no tienen conocimiento de los principales costos asociados a los servicios de salud que brinda la unidad hospitalaria, como se muestra en la figura 4.
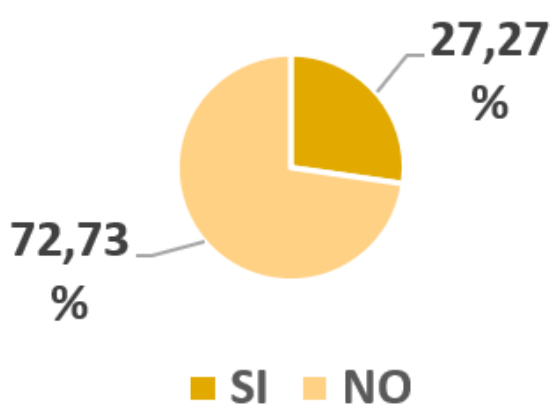

Figura 4. Conocimiento de principales costos de servicios de salud

La población encuestada menciona los principales obstáculos detectados por lo que no se utilizan las herramientas de contabilidad de costos o gestión en unidades de salud pública:

1. Falta de personal

2. Gran cantidad de servicios

3. No contar con sistemas adecuados para la aplicación

4. Falta de conocimiento en contabilidad de costos

5. Alta demanda dificulta tener la información de todo el proceso

6. Falta de información
7. Falta de cultura institucional

8. Cambio constante de personal

9. Dificultad de recolección de información

Para garantizar la integralidad en la atención de salud a la población ecuatoriana cuando no existe capacidad resolutiva dentro de la institución, según la percepción de los encuestados los principales motivos por el que se transfiere o deriva un paciente a otra unidad de salud se exponen en la tabla 1.

Tabla 1

Principales motivos de transferencia o derivación de un paciente según percepción directivos.

\begin{tabular}{|c|c|c|c|c|c|c|c|}
\hline & \multicolumn{5}{|c|}{ Porcentaje orden de prioridad } & Media & \\
$\begin{array}{c}\text { Principales } \\
\text { motivos }\end{array}$ & 1 & 2 & 3 & 4 & 5 & $\begin{array}{l}\text { Orden de } \\
\text { prioridad } \\
\text { final }\end{array}$ \\
\hline $\begin{array}{c}\text { Necesidad } \\
\text { de otro nivel } \\
\text { de atención }\end{array}$ & 41,67 & 33,33 & 25,00 & - & - & 1,83 & 1 \\
\hline Falta Equipo & 41,67 & 16,67 & 25,00 & 8,33 & 8,33 & 2,25 & 2 \\
\hline $\begin{array}{c}\text { Falta de } \\
\text { espacio } \\
\text { físico }\end{array}$ & 16,67 & 16,67 & 50,00 & 8,33 & 8,33 & 2,75 & 3 \\
\hline $\begin{array}{c}\text { Falta de } \\
\text { Insumo }\end{array}$ & 8,33 & 16,67 & 8,33 & 33,33 & 33,33 & 3,67 & 4 \\
\hline $\begin{array}{c}\text { Falta de } \\
\text { Especialista }\end{array}$ & - & 16,67 & - & 41,67 & 41,67 & 4,08 & 5 \\
\hline
\end{tabular}

Fuente: Tomado de la encuesta a responsables de áreas administrativas Hospital Público Segundo Nivel de Salud Manabí.

Con base a la información recopilada se obtuvo la cantidad de pacientes y los principales motivos por los que se transfiere o deriva un paciente a otra unidad de salud en la Zona 4 Manabí-Santo Domingo período 2019, ver tabla 2.

Tabla 2

Cantidad de pacientes y principales motivos de transferencia o derivación

\begin{tabular}{|c|c|}
\hline Año 2019 & \\
$\begin{array}{c}\text { Motivo derivación/ } \\
\text { transferencia }\end{array}$ & Cantidad de pacientes \\
\hline
\end{tabular}




\begin{tabular}{|c|c|}
\hline Falta de equipamiento & 12.372 \\
\hline Falta de insumo & 9.253 \\
\hline $\begin{array}{c}\text { Necesidad otro nivel de } \\
\text { atención }\end{array}$ & 4.123 \\
\hline $\begin{array}{c}\text { Tratamiento oncológico } \\
\text { integral }\end{array}$ & 1.142 \\
\hline Falta de especialista & 1.346 \\
\hline Falta de espacio físico & 141 \\
\hline Problemas de infraestructura & 19 \\
\hline Total & 28.396 \\
\hline
\end{tabular}

Fuente: Tomado de la Coordinación Zonal 4 Salud Manabí-Santo Domingo

El deficiente manejo de los costos y el desconocimiento de las herramientas que proporciona la contabilidad de gestión para la toma de decisiones es el punto de partida para proponer un procedimiento que facilita este proceso y permite su aplicación ante diferentes alternativas adaptables a las decisiones, utilizando como base los costos, de brindar un servicio de integral o comprarlo a prestadores externos privados, este procedimiento puede ser aplicado por los administradores o directivos para las decisiones que correspondan ante una problemática existente.

Tabla 3

Fases, pasos, objetivos para la toma de decisiones

\begin{tabular}{ccc}
\hline Fases & Pasos & Objetivos \\
\hline $\begin{array}{c}\text { 1ra fase } \\
\text { informativa }\end{array}$ & $\begin{array}{c}\text { Obtener información } \\
\text { respecto a las derivaciones } \\
\text { de los servicios integrales } \\
\text { de salud, datos en torno a } \\
\text { los motivos de derivación, } \\
\text { costos de estas alternativas, } \\
\text { identificar el flujo del } \\
\text { servicio de salud. }\end{array}$ & $\begin{array}{c}\text { Obtener los datos } \\
\text { disponibles relacionados } \\
\text { con la información tanto } \\
\text { externa como interna, } \\
\text { asíno las variables, } \\
\text { tanto cualitativas como } \\
\text { cuantitativas, relevantes } \\
\text { asociados al problema en } \\
\text { cuestión. }\end{array}$ \\
$\begin{array}{c}\text { 2da fase } \\
\text { del problemanto }\end{array}$ & $\begin{array}{c}\text { Generar todas las } \\
\text { alternativas posibles de } \\
\text { solución: }\end{array}$ & $\begin{array}{c}\text { Conocer las } \\
\text { circunstancias que rodean } \\
\text { el problema o de una } \\
\text { situación similar para } \\
\text { seleccionar un curso de }\end{array}$ \\
& $\begin{array}{c}\text { Desarrollar un sistema favorable. } \\
\text { contable para el registro, } \\
\text { control de recursos y } \\
\text { costos. }\end{array}$ &
\end{tabular}

Determinar las necesidades de compas más eficientes.

3ra Fase Análisis de costos de las alternativas

4ta fase Selección de alternativa

5 ta fase Implantación

6ta fase: medición de la efectividad y mejora

Determinar los diferentes indicadores y comparar los resultados. curso de acción alternativo tomando la decisión más eficiente para la unidad de salud.

Fuente: Adaptado del modelo para la toma de

Elaboración: Autoría Propia
Analizar el comportamiento de los costos asociados a las alternativas de hacer o comprar el servicio de salud integral.

Elegir una de las alternativas propuestas, para esto el administrador puede apoyarse en las herramientas de y hagan un seguimiento de las consecuencias, costos y oportunidades proporcionadas por cada una de las alternativas.

En esta etapa el administrador lleva la decisión a la acción y da su informe sobre el Se debe monitorear la implementación de las alternativas

Evaluar la efectividad de la decisión tomada en el desempeño de la organización. información que calculen progreso y la solución.

\section{decisiones propuesto por Garza R. (2007).}




\section{Discusión}

A partir de los hallazgos encontrados llama la atención el alto porcentaje desconocimiento de los directivos del área hospitalaria, sobre la existencia de herramientas de contabilidad de gestión o costos e incluso de los principales servicios que brinda la unidad de salud y de los rubros que lo componen, así como la posibilidad que estas proporcionan para la mejora en la toma de decisiones, control de los recursos e incremento de la eficiencia.

El control de gestión en una organización pública es clave, ya que proporciona información para analizar desviaciones y las posibles medidas de retroalimentación, lo que implica pasar de "cuánto" se gasta a "cómo" se gasta (López, 2015).

Los esfuerzos de investigación en contabilidad de gestión en Iberoamérica son destacados por España, Brasil, Venezuela y México también han impulsado las tendencias emergentes en contabilidad de gestión en los países diferentes a España, que intenta consolidarse en algunos casos y es casi inexistente en otros (Salgado Castillo, 2011). Se da el caso en Ecuador específicamente el sector de salud pública en la Zona 4 Manabí-Santo Domingo, donde se identifica carencia de personal capacitado sobre la temática para ejecutar planes de acción en base a esta herramienta, como lo muestra los resultados en la figura 1,2 y 3 .

Los presupuestos pasaban a ser las herramientas de gestión más utilizadas (Falguera \& Amat Salas, 2002). La figura 1 muestra un $8,33 \%$ que si conoce herramientas de contabilidad de gestión corresponde al único funcionario de los doce encuestados, quien especificó el uso de presupuestos en la institución de salud.

La unidad de salud deberá evaluar los beneficios que aporta la implementación de herramientas de contabilidad de gestión o costos, si son mayores que los gastos de recursos de los supuestos obstáculos que expone la tabla 1.

La contabilidad de gestión pretende aportar información para adoptar decisiones de forma racional, con el menor riesgo posible. Pretende servir de orientación o base de referencia para todo tipo de decisiones internas de la empresa (Falguera \& Amat Salas, 2002). Al realizar el análisis en la tabla 2 y tabla 3 sobre los principales motivos de transferencia o derivación de un paciente, se puede contrastar la percepción de los directivos con la realidad. Según la percepción el primer motivo derivación o transferencia de un paciente es necesidad de otro nivel de atención y la existente es falta de equipamiento. Adicional ninguno de los demás motivos coincide de acuerdo al orden de prioridad.

La aplicación de sistemas de contabilidad de gestión es un gran soporte y herramienta gerencial en el área hospitalaria, sin embargo, en la práctica tiene poca relevancia por desconocimiento por parte de los directivos hospitalarios de los beneficios en la gestión al aplicar estas herramientas, los principales productos hospitalarios y asumir por percepción sin estudiar en base a información la realidad de la unidad de salud.

La optimización de los recursos evaluando y controlando el costo por paciente, no solo permite el logro de eficiencia en los procesos sino ampliar la cobertura de servicios de salud, para ponerlo en marcha es necesario personal capacitado para la obtención de resultados confiables y oportunos que sean de gran utilidad para los directivos en la elaboración de políticas de salud y solicitudes de asignaciones presupuestarias.

El sistema de información de costos debe ser integrador, porque debe existir una apropiada coordinación entre departamentos, iniciando con el de planificación y todos los involucrados en la toma de decisiones.

\section{Referencias bibliográficas}

Constitución de la República del Ecuador. (20 de Octubre de 2008). OAS. Recuperado el 2020 de Junio de 21, de https://www.oas. org/juridico/pdfs/mesicic4_ecu_const.pdf

INEC. (11 de Noviembre de 2016). INEC. 
Recuperado el 21 de Junio de 2020, de https://www.ecuadorencifras.gob.ec/ crece-la-participacion-del-sector-saluden-la-economia-ecuatoriana/

Ministerio de Salud Pública. (10 de Abril de 2015). Ministerio de Salud Pública. Recuperado el 21 de Junio de 2020, de http://instituciones.msp.gob.ec/ images/Documentos/subse_gobernanza/ Convenio_RPIS\%202015.pdf

Vargas, V., \& Hernández, C. (15 de Julio de 2009). Sistemas de Información de costos para la gestión hospitalaria. Revista de Ciencias Sociales. Recuperado el 21 de Junio de 2020, de http://ve.scielo. org/scielo.php?script $=$ sci_arttext\&pid $=\mathrm{S} 1315-95182009000400012$

Falguera, M., \& Amat Salas, O. (2002). La contabilidad de gestión en los centros sanitarios. Universitat Pompeu Fabra. Recuperado el 14 de Julio de 2020, de https://www.tdx.cat/handle/10803/7332

López, M. (2015). COST IN THE PUBLIC SECTOR. EKOTEMAS 1(1), 1-10. Recuperado el 15 de Julio de 2020, de http://www.ekotemas.cu/index.php/ ekotemas/article/download/93/22

Salgado Castillo, J. (2011). Tendencias de investigación en contabilidad de gestión en Iberoamérica (1998-2008). Cuadernos de Contabilidad, 12(30), 273-305. Recuperado el 14 de Julio de 2020, de http:// www.scielo.org.co/scielo.php?script $=$ sci arttext\&pid=S0123-14722011000100011

Garza Ríos, Rosario, \& González Sánchez, Caridad, \& Salinas Gómez, Eivis (2007). TOMA DE DECISIONES EMPRESARIALES: UN ENFOQUE MULTICRITERIO MULTIEXPERTO. Ingeniería Industrial, XXVIII(1),29-36. [fecha de Consulta 13 de Agosto de 2020]. ISSN: 0258-5960. Disponible en: https://www.redalyc.org/articulo. oa?id=3604/360433562007 